\title{
Case series: Zonulectomy in the treatment of aqueous misdirection
}

\author{
Ashraf A Khan*, Harry Bennett and Hilary Devlin \\ Princess Alexandra Eye Pavilion, Edinburgh, United Kingdom
}

\begin{abstract}
Purpose: The management for aqueous misdirection remains a challenge and numerous strategies have been described. Unfortunately the failure rate for medical and laser therapy is high. Previous reports describe a technique of combined vitrectomy with hyaloido-zonulectomy for the treatment of aqueous misdirection. We report our case series of patients managed with a modified version of this technique.

Methods: Seven consecutive cases of aqueous misdirection diagnosed between January 2006 and September 2010 (7 eyes of 5 patients) were included in the study. Five eyes underwent pars plana vitrectomy combined with peripheral zonulectomy, two patients underwent a peripheral zonulectomy from an anterior approach. Outcome measures included BCVA, IOP, medications and slit lamp findings.

Results: All patients responded to surgery with reduction in IOP and deepening of the AC. One patient needed an additional proceedure after 4 months and one patient suffered from post operative endophthalmitis. Mean follow up 27 months (range 14-42 months).

Conclusions: A reason for the high recurrence rate in patients who have had vitrectomy alone is due to difficulty in establishing unobstructed flow from the vitreous cavity to the anterior chamber. Our case series highlights the importance of the zonulectomy which we feel to be a crucial step as it establishes the unobstructed communication.

Three port pars plana vitrectomy combined with hyaloido-zonulectomy is an effective treatment for the management of aqueous misdirection; experienced anterior segment surgeons may consider performing a peripheral iridectomy and zonulectomy from an anterior approach.
\end{abstract}

\section{Introduction}

Aqueous misdirection is a rare condition thought to be caused by aqueous being diverted posteriorly into the vitreous cavity [1]. It most commonly occurs after glaucoma filtration surgery but can occur after cataract extraction, laser iridotomy, capsulotomy, miotic therapy and cyclophotocoagulation [2-6]. It is more common in patients who have a history of treatment for angle closure glaucoma [7].

It is characterised by central and peripheral shallowing of the anterior chamber (AC) with a patent iridotomy and no evidence of suprachoroidal haemorrhage or effusion $[8,9]$. The management for aqueous misdirection remains a challenge and numerous strategies have been described. Medical therapies with cycloplegics and aqueous suppressants, laser therapies with peripheral iridotomy and anterior hyaloidotomy and surgical therapy with pars plana vitrectomy have all been described [2,10-12]. Unfortunately the failure rate for medical and laser therapy is high. Previous reports describe a technique of combined vitrectomy with hyaloido-zonulectomy for the treatment of aqueous misdirection [13]. We report our case series of patients managed with a modified version of this technique, and in some cases performed some time after diagnosis of aqueous misdirection.

\section{Methods}

7 consecutive cases of aqueous misdirection diagnosed between January 2006 and September 2010 (7 eyes of 5 patients) were included in the study. 5 eyes underwent pars plana vitrectomy combined with peripheral hyaloido-zonulectomy, two patient underwent a peripheral zonulectomy from an anterior approach.
Surgical technique: patients underwent 3 port pars plana core vitrectomy (3PPV). Following this, the vitrector was then angled upwards to perform a hyaloido-zonulectomy and a peripheral iridectomy from a posterior approach.

Two patients underwent a zonulectomy and peripheral iridectomy from an anterior approach. Here the AC was deepened with viscoelastic and an AC maintainer was used. From a limbal incision the vitrector was angled backwards through the previous peripheral iridectomy to initially enlarge it and then maneuvered further posteriorly to perform a zonulectomy as well.

\section{Results}

See summary table 1.

\section{Case 1}

71 year old female diagnosed with chronic angle closure glaucoma (CACG) underwent uncomplicated trabeculectomy surgery. Her low pre-op intra ocular pressure (IOP) was only maintained with oral acetazolomide medication. 2 weeks post op she was noted to have

Correspondence to: Dr. Ashraf Khan, Speciality Registrar, Princess Alexandra Eye Pavilion, Chalmers Street, Edinburgh, EH3 9HA, United Kingdom, Tel: +44 131536 1000; Fax: +44 131536 3897; E-mail: Ashraf.khan@nhs.net

Key words: aqueous misdirection, zonulectomy, hyaloido-zonulectomy, vitrectomy Received: March 23, 2016; Accepted: April 12, 2016; Published: April 15, 2016 


\begin{tabular}{|c|c|c|c|c|c|c|c|c|c|c|c|}
\hline \multirow[b]{2}{*}{ Case } & \multirow[b]{2}{*}{ Details } & \multirow[b]{2}{*}{ Eye } & \multirow[b]{2}{*}{ Diagnosis } & \multirow[b]{2}{*}{ Surgery } & \multicolumn{2}{|c|}{ IOP } & \multicolumn{2}{|c|}{ BCVA } & \multicolumn{2}{|l|}{ Treatment } & \multirow{2}{*}{$\begin{array}{r}\text { Follow up } \\
\text { (months) }\end{array}$} \\
\hline & & & & & Pre & Post & Pre & Post & Pre & Post & \\
\hline 1 & $71 \mathrm{~F}$ & Right & CACG & Trab & 12 & 11 & $6 / 6$ & $6 / 9$ & Latanoprost, timolol \& Acetazolomide SR 250mg BD & duotrav & 28 \\
\hline 2 & $30 \mathrm{M}$ & Right & plateau iris syndrome & Trab & 24 & 20 & $6 / 9$ & $6 / 5$ & cosopt, latanoprost, brimonidine and acetazolomide $250 \mathrm{mg} \mathrm{BD}$ & timolol & 42 \\
\hline \multirow[t]{2}{*}{3} & $77 \mathrm{~F}$ & Right & CACG & Trab & 33 & 18 & $6 / 12$ & $6 / 12$ & cosopt & duotrav & 33 \\
\hline & & Left & OHT and shallow AC & Phaco & 23 & 15 & $6 / 12$ & $6 / 9$ & timolol & duotrav & 36 \\
\hline \multirow[t]{2}{*}{4} & $44 \mathrm{~F}$ & Right & plateau iris syndrome & Trab & 17 & 14 & $6 / 5$ & $6 / 5$ & Acetazolomide SR 250 OD & 0 & 14 \\
\hline & & Left & plateau iris syndrome & Phaco-Trab & 14 & 12 & $6 / 6$ & $6 / 18$ & Acetazolomide SR 250 OD & 0 & 14 \\
\hline 5 & $79 \mathrm{~F}$ & Left & CACG & Trab & 32 & 10 & $6 / 12$ & $6 / 24$ & Acetazolomide SR 250 BD & timolol & 22 \\
\hline
\end{tabular}

$\mathrm{F}=$ female, $\mathrm{M}=$ male, $\mathrm{CACG}=$ chronic angle closure glaucoma, $\mathrm{OHT}=$ ocular hypertension, $\mathrm{AC}=$ anterior chamber, Trab= Trabeculectomy, $\mathrm{Phaco}=\mathrm{Phacoemulsification}$ of lens

a raised IOP of 34 for which she underwent laser suture lysis and bleb needling with 5 fluro-uracil. She was intolerant to atropine and underwent YAG capsulotomy and to the anterior hyaloid face 6 months post op. Her IOP remained high $(28 \mathrm{mmHg})$ and she underwent a 3PPV combined with hyaloido-zonulectomy. She has since been maintained on two topical agents and her latest IOP was 11, two and a half years after the vitrectomy.

\section{Case 2}

A 31 year old male with plateau iris syndrome underwent difficult trabeculectomy surgery. During the surgery it was noted that the globe was tense for which he required intravenous mannitol. The iris protruded through the sclerostomy and miochol was used. At one week his IOP was $26 \mathrm{mmHg}$ with a very shallow $\mathrm{AC}$ and he was diagnosed with aqueous misdirection. He underwent $3 \mathrm{PPV}$ with subsequent deepening of the AC. Following this he was controlled with atropine but developed an intolerance. After 18 months on stopping atropine his AC was very shallow and his IOP was $52 \mathrm{mmHg}$. He underwent a further vitrectomy combined with phacoemulsification of the lens but his pressure remained high at 30 . His AC progressively shallowed and his pressure increased to 44 . He underwent an iridectomy, zonulectomy and anterior vitrectomy from an anterior approach with immediate deepening of the anterior chamber. His post op IOP was 18 and despite maintaining a deep AC his IOP increased to $40 \mathrm{mmHg}$ after four months and he underwent revision of his peripheral iridectomy and anterior hyloidectomy from a posterior approach. His IOP post op was $20 \mathrm{mmHg}$ and has been maintained for four years on just one topical agent.

\section{Case 3}

A 77 year old female with CACG in the right eye and ocular hypertension with a shallow AC in the left, underwent trabeculectomy surgery to the right eye which had previously undergone cataract surgery. Aqueous misdirection was diagnosed two months post op. She developed an intolerance to atropine and her IOP steadily rose over three years when she underwent 3PPV combined with hyaloidozonulectomy. Her IOP has been controlled at 18 on two topical drops for three years. Over the same time period she underwent uncomplicated cataract extraction (phacoemulsification) in her left eye. Two years following this she was noted to have a shallow $\mathrm{AC}$ and raised IOP at 32 $\mathrm{mmHg}$. She underwent $3 \mathrm{PPV}$ and hyaloido-zonulectomy four months later. Her IOP has been controlled for the last four years at 15 on two topical drops.

\section{Case 4}

A 44 year old female with plateau iris syndrome and previous bilateral laser peripheral iridotomies underwent a right trabeculectomy.
Her preop IOP was $17 \mathrm{mmHg}$ controlled on acetazolomide tablets. On day one post op she was noted to have a pressure of $24 \mathrm{mmHg}$ and was diagnosed with aqueous misdirection. She was controlled on acetazolomide $250 \mathrm{mg}$ QDS and underwent a 3PPV and hyaloidozonulectomy the following week. Her IOP following this was controlled at $12 \mathrm{mmHg}$ on no topical treatment, She remained on acetazolomide to control the left IOP. Later the same month, the patient underwent a left phaco-trabeculectomy. Aqueous misdirection was diagnosed on day one post op again, with an IOP of $12 \mathrm{mmHg}$. She underwent a vitrectomy and hyaloido-zonulectomy later the same day. Unfortunately her post op recovery was complicated by endophthalmitis where her vision reduced to hand movements in the affected eye. She underwent a further vitrectomy with intra-vitreal antibiotics with slow resolution of the endophthalmitis. Her latest IOP is $14 \mathrm{mmHg}$ in her right eye and $12 \mathrm{mmHg}$ in her left after one year and she is on no treatment. The vision in the left eye has reduced from $6 / 6$ preop to $6 / 18$.

\section{Case 5}

A 79 year old female with narrow angle glaucoma underwent an uncomplicated trabeculectomy to her left eye. One year later she underwent a trabeculectomy to her right eye complicated by aqueous misdirection diagnosed one week post op. She underwent a 3PPV and subsequent bleb needling two weeks later. Six months later she underwent an uncomplicated phacoemulsifiaction to her left cataract and was followed up six monthly for five years. At this point she had an elevated IOP at $22 \mathrm{mmHg}$ with a slightly shallow AC. She was treated initially with atropine drops which deepened her AC but she continued to have a raised IOP at $30 \mathrm{mmHg}$. The pressure was controlled with oral acetazolomide SR $250 \mathrm{mg}$ twice daily and two topical agents. Given the deep AC her signs were not typical of aqueous misdirection and she underwent a redo trabeculectomy. One week post op the AC was very shallow and she was diagnosed with aqueous misdirection. After no resolution after one week she under went a left peripheral iridectomy, anterior vitrectomy and zonulectomy from an anterior approach. Her AC deepened dramatically on the table and remained deep throughout her post operative recovery. For the last two years her she has not had any recurrence. Her latest IOP was $12 \mathrm{mmHg}$ in the right and $10 \mathrm{mmHg}$ in the left.

\section{Discussion}

Aqueous misdirection results from posterior flow of aqueous into the vitreous cavity. Although the mechanism is not fully understood, a number of possibilities have been suggested such as anterior rotation of the ciliary body causing ciliolenticular touch and ciliary block, as well as abnormalities in the choroid, lens, zonules and vitreous $[8,13]$.

By creating a communication between the anterior chamber and 
vitreous cavity, aqueous flow can be re-established to the anterior chamber. Medical management in aqueous misdirection consists of cycloplegia and topical and/or oral IOP lowering agents. In some pseudophakic patients, YAG laser capsulotomy with hyaloidotomy is an option as this creates a channel for fluid to flow to the anterior chamber once the anterior hyaloid face has been disrupted. Unfortunately, a number of patients do not respond to the treatment and in those that do, there is a high relapse rate $[10,13]$.

Other options include trans-scleral cyclodiode and argon laser iridoplasy which attempts to shrink the processes and thus break the ciliary block $[14,15]$. A total pars plana vitrectomy can facilitate the flow of aqueous into the anterior chamber by disrupting the anterior hyaloid face however this may not be enough to break the cycle. One of the reasons for a high recurrence rate in patients who have had vitrectomy alone is due to the difficulty in establishing unobstructed flow from the vitreous cavity to the anterior chamber by removing the anterior hyaloid face $[1,3]$. In our case series most patients underwent a standard three port pars plana vitrectomy combined with hyaloidozonulectomy through the pre existing peripheral iridotomy. This we feel to be the most important step in the procedure as it establishes the unobstructed communication. Two patients underwent a zonulectomy from an anterior approach, with immediate visible deepening of the $\mathrm{AC}$, further suggesting that this step is the crucial in allowing the aqueous to flow into the $\mathrm{AC}$ and breaking the cycle. Unfortunately one of these patients required an extra surgery, suggesting an adequate sized peripheral iridectomy and zonulectomy is required to achieve a satisfactory outcome.

Three port pars plana vitrectomy combined with hyaloidozonulectomy is an effective treatment for the management of aqueous misdirection; experienced anterior segment surgeons may consider performing a peripheral iridectomy and zonulectomy from an anterior approach as a possible management option.

\section{References}

1. Debrouwere V, Stalmans P, Van Calster J, Spileers W, Zeyen T, et al. (2012) Outcomes of different management options for malignant glaucoma: a retrospective study. Graefes Arch Clin Exp Ophthalmol 250: 131-141. [Crossref]

2. HarbourJW, Rubsamen PE, Palmberg P (1996) Pars planavitrectomy in the managemen of phakic and pseudophakic malignant glaucoma. Arch Ophthalmol 114: 1073-1078. [Crossref]

3. Byrnes GA, Leen MM, Wong TP, Benson WE (1995) Vitrectomy for ciliary block (malignant) glaucoma. Ophthalmology 102: 1308-1311. [Crossref]

4. Cashwell LF, Martin TJ (1992) Malignant glaucoma after laser iridotomy Ophthalmology 99: 651-658. [Crossref]

5. Mastropasqua L, Ciancaglini M, Carpineto P, Lobefalo L, Gallenga PE (1994) Aqueous misdirection syndrome: a complication of neodymium: YAG posterior capsulotomy. $J$ Cataract Refract Surg 20: 563-565. [Crossref]

6. Lynch MG, Brown RH, Michels RG, Pollack IP, Stark WJ (1986) Surgicalvitrectomy for pseudophakic malignant glaucoma. Am J Ophthalmol 102: 149-153. [Crossref]

7. Quigley HA (2009) Angle-closure glaucoma-simpler answers to complex mechanisms: LXVI Edward Jackson Memorial Lecture. Am J Ophthalmol 148: 657-669. [Crossref]

8. Luntz MH, Rosenblatt M (1987) Malignant glaucoma. SurvOphthalmol 32: 73-93. [Crossref]

9. Ruben S, Tsai J, Hitchings RA (1997) Malignant glaucoma and its management. $B r J$ Ophthalmol81: 163-167. [Crossref]

10. Chandler PA, Simmons RJ, Grant WM (1968) Malignant glaucoma. Medical and surgical treatment. Am J Ophthalmol 66: 495-502. [Crossref]

11. Simmons RJ (1972) Malignant glaucoma. Br J Ophthalmol 56: 263-272. [Crossref]

12. Sharma A, Sii F, Shah P, Kirkby GR (2006) Vitrectomy-phacoemulsificationvitrectomy for the management of aqueous misdirection syndromes in phakic eyes. Ophthalmology 113: 1968-1973. [Crossref]

13. Bitrian E, Caprioli J (2010) Pars plana anterior vitrectomy, hyaloido-zonulectomy, and iridectomy for aqueous humor misdirection. Am J Ophthalmol 150: 82-87. [Crossref]

14. Herschler J (1980) Laser shrinkage of the ciliary processes. A treatment for malignant (ciliary block) glaucoma. Ophthalmology 87: 1155-1159. [Crossref]

15. Stumpf TH, Austin M, Bloom PA, McNaught A, Morgan JE (2008) Transscleralcyclodiode laser photocoagulation in the treatment of aqueous misdirection syndrome. Ophthalmology 115:2058-2061. [Crossref]

Copyright: (C2016 Khan AA. This is an open-access article distributed under the terms of the Creative Commons Attribution License, which permits unrestricted use, distribution, and reproduction in any medium, provided the original author and source are credited. 